\title{
Gold-standard diagnostics are tarnished by lytic bacteriophage
}

Running Header: Lytic vibriophage reduce Vibrio cholerae detection

E.J. Nelson ${ }^{1,2 \dagger, *}$, J.A. Grembi ${ }^{3,4 \dagger}$, D.L. Chao ${ }^{5}$, J.R. Andrews ${ }^{3}$, L. Alexandrova ${ }^{6}$, P. H. Rodriguez ${ }^{1}$, V.V. Ramachandran ${ }^{2}$, Md.A. Sayeed ${ }^{1}$, W. J. Wamala ${ }^{7}$, A.K. Debes ${ }^{8}$, D.A. Sack ${ }^{8}$, A. J. Hryckowian $^{9}$, F. Haque ${ }^{10 \dagger, 11}$, S. Khatun ${ }^{10 \dagger}$, M. Rahman ${ }^{10 \dagger, 12}$, A. Chien ${ }^{6}$, A.M. Spormann ${ }^{4}$, G.K. Schoolnik ${ }^{3}$

${ }^{1}$ Departments of Pediatrics and Environmental and Global Health, University of Florida (USA)

${ }^{2}$ Department of Pediatrics, School of Medicine, Stanford University (USA)

${ }^{3}$ Department of Medicine, School of Medicine, Stanford University (USA)

${ }^{4}$ Department of Civil and Environmental Engineering, Stanford University (USA)

${ }^{5}$ Institute for Disease Modeling (USA)

${ }^{6}$ Vincent Coates Foundation Mass Spectrometry Laboratory, Stanford University (USA)

${ }^{7}$ Country Preparedness and IHR (CPI), World Health Organization (South Sudan)

${ }^{8}$ Johns Hopkins Bloomberg School of Public Health, Johns Hopkins University (USA)

${ }^{9}$ Department of Microbiology, School of Medicine, Stanford University (USA)

${ }^{10}$ Institute of Epidemiology, Disease Control and Research, Ministry of Health and Family Welfare, Government of Bangladesh. (Bangladesh)

${ }^{11}$ University College London (UK)

${ }^{12}$ International Centre for Diarrhoeal Disease Research, Bangladesh. (Bangladesh)

† Former institution

*Address correspondence to Eric J. Nelson, MD PhD, eric.nelson@ufl.edu

Abstract: 189 words

Main text: 2344 words 


\section{ABSTRACT}

2 Background. A fundamental clinical and scientific concern is how lytic bacteriophage, as well as

3 antibiotics, impact diagnostic positivity.

4 Methods. Cholera was chosen as a model disease to investigate this important question.

5 Patients with diarrheal disease were enrolled at two remote hospitals in Bangladesh. Diagnostic

6 performance was assessed as a function of lytic bacteriophage detection, as well as exposure

7 to the first-line antibiotic azithromycin detected by mass spectrometry.

8 Results. Among diarrheal samples positive by nanoliter quantitative PCR for Vibrio cholerae

$9(n=78 / 849)$, the odds that a rapid diagnostic test (RDT) or qPCR was positive was reduced by

$1089 \%(\mathrm{OR} 0.108 ; 95 \% \mathrm{Cl} 0.002-0.872)$ and $87 \%$ (OR $0.130 ; 95 \% \mathrm{Cl} 0.022-0.649)$ when lytic

11 bacteriophage were detected, respectively. The odds that a rapid diagnostic test (RDT) or qPCR

12 was positive was reduced by more than 99\% (OR $0.00 ; 95 \% \mathrm{Cl}: 0.00-0.28)$ and $89 \%(\mathrm{OR} 0.11$;

$1395 \% \mathrm{Cl}:$ 0.03-0.44) when azithromycin was detected, respectively.

14 Conclusions. Estimations of cholera burden may improve by accommodating for the negative

15 effect of antimicrobial exposure on diagnostic positivity. Furthermore, the findings herein

16 challenge our current approach to interpreting and developing bacterial diagnostics given

17 variable rates of lytic bacteriophage and antibiotic exposure. 


\section{BACKGROUND}

19 There are approximately 4.5 billion diarrheal diseases cases per year [1]. While the 2-4 million

20 cases of cholera that occur annually represent a small fraction of the total cases [2], cholera

21 inflicts high morbidity and mortality on populations with extreme poverty. Outbreaks begin when

22 immunologically susceptible human hosts are exposed to the Gram-negative pathogen $V$.

23 cholerae (O1 and 0139 serogroups) from contaminated food or water [3]. Before modern

24 rehydration regimens, mortality rates rose above $20 \%$ [4] from acute secretory diarrhea resulting

25 from the action of cholera toxin [3]. Treated with oral or intravenous rehydration, mortality rates

26 decrease to less than one percent $[5,6]$. Antibiotics are recommended for cholera patients with

27 moderate to severe dehydration [7, 8], but in practice most cholera patients are likely ordered

28 antibiotics. Asymptomatic cases are detected by a rise in antibody titer but negative stool

29 studies [9]. Recovered patients become susceptible over time as a function of the durability of

30 their immune response [3]. V. cholerae are shed from the human host with increased infectivity

$31[10,11]$. This 'hyper-infectivity' is suggested to drive the exponential phase of outbreaks [12].

32 Patients can shed V. cholerae specific lytic bacteriophage (ICP1, 2, 3); ICP1 is specific for O1

33 V. cholerae $[13,14]$. These vibriophages are proposed to quench outbreaks based on data that

34 a higher percentage of patients shed vibriophage during the collapse of an outbreak [15-17].

35 Diagnostically, culture and PCR approaches are the best available 'gold-standards' for the

36 detection of $V$. cholerae $[18,19]$. Alternative methods include direct immuno-fluorescence

37 microscopy for the O-antigen polysaccharide (OPS) [20], RDTs that rely on OPS specific

38 antibodies, and recently by nl-qPCR [21, 22].

39 The rationale for this study was based on the recognition that cholera RDTs have limited

40 adoption because of variable performance for unknown reasons [19, 23-26]; immediately testing

41 stool samples demonstrated broad sensitivities (58-100\%) and specificity (71-100\%). A modified 
42 method enriches for $V$. cholerae in alkaline peptone water (APW) to increase specificity to 91-

$4399 \%$ yet is associated with a decrease in sensitivity [23, 24, 27]. Both lytic phage and antibiotics

44 have been postulated to impact diagnostics [26]. Using cholera as a model, we tested the

45 hypothesis that lytic bacteriophage, and antibiotics, negatively impact diagnostics within the

46 confines of a previously published clinical study [28]. In brief, the study was conducted from

47 September to December 2015 at a district and sub-district hospital in the remote Northern

48 district of Netrokona that is prone to seasonal cholera outbreaks. Inclusion criteria were patients

49 at least two-months old and presented with acute ( $<7$ days) diarrhea ( $>3$ loose stools in the 24

50 hours prior to admission) without complications.

\section{METHODS}

\section{Subjects}

53 This study was conducted with the confines of previously published studies in Bangladesh [28]

54 and South Sudan [29]. Ethical approvals were obtained for the Bangladesh study at the

55 Institutional Review Boards (IRBs) of Stanford University School of Medicine and the Institute of

56 Epidemiology, Disease Control and Research, Bangladesh Ministry of Health and Family

57 Welfare [28], and for South Sudan Study at the IRBs of Johns Hopkins Bloomberg School of

58 Public Health and the South Sudan Ministry of Health, Directorate of Monitoring, Evaluation and

59 Research [29]. Written informed consent was obtained from participants over 18 years, or

60 guardians of participants.

\section{Clinical Study}

62 In Bangladesh, inclusion criteria were patients at least two-months of age presenting with acute

63 (< 7 days) diarrhea (>3 loose stools in the prior 24 hours) without clinical complications. Sample

64 collection occurred from September to December 2015 at a district and sub-district hospital in

65 the remote Northern district of Netrokona that is prone to seasonal cholera outbreaks. In South

66 Sudan, inclusion criteria were patients presenting at a cholera treatment center in Juba who 
67 were at least 6 months-old, had diarrhea (>3 loose stools in the prior 24 hours) and no history of

68 cholera vaccination. Samples were collected from August to September 2015.

\section{Laboratory Procedures}

70 For samples collected in Bangladesh, the methods have been previously described [21, 22]. In

71 brief, the first stool sample voided was collected immediately after admission to avoid exposure

72 to hospital administered antibiotics. The supernatants from V. cholerae positive stools were

73 tested for antibiotic exposure using a LC/MS protocol for a 1100 series HPLC (Agilent

74 Technologies) integrated with an LTQ XL ion trap mass spectrometer (Thermo Fisher Scientific)

75 [21]. The stool samples were tested by RDT (Crystal VC, Span Diagnostics) after enrichment in

76 APW for 6 hours or overnight [28]. The first and last samples collected per day were stored in

77 Cary-Blair media $\left(4^{\circ} \mathrm{C}\right)$ for culture at a central reference laboratory in Dhaka (icddr,b); samples

78 were stored for up to 1 month. Aliquots $(500 \mu \mathrm{l})$ from all patients were stored in $1.3 \mathrm{ml}$ RNAlater

79 (Invitrogen).

80 For Bangladesh samples, DNA was extracted using the MoBio Power Soil 96-well plate system

81 (Qiagen; formerly PowerSoil). DNA extracts were screened in technical replicates for V.

82 cholerae by qPCR in a 384-well Light Cycler (Roche) using tcp $A^{\text {set1 }}$ primers (Table S1) [21].

83 Samples that had CT values less than 25 were defined as positive. Samples with CT values

84 from 25 to 31 were evaluated by PCR for ompW [8]. In parallel, nl-qPCR was performed in

85 technical replicates with $t c p A^{\text {set1 }}$ and additional targets [21, 22]. Cyber Green master mix (Sigma

86 Aldrich) was used for both qPCR and nl-qPCR however there was 1.8-fold more DNA in nl-

87 qPCR reactions. Cycle thresholds for positivity for qPCR and nl-qPCR were 29 and 28,

88 respectively. $16 \mathrm{~S}$ rDNA analysis utilized previously published methods and data [21] on nl-

89 qPCR V. cholerae positive samples for tcpA (Table S1). Lytic vibriophages ICP1, 2, and 3 were

90 detected by PCR (Table S1). For samples collected in South Sudan, analyses for V. cholerae 
91 have been previously described on DNA extracted from dried stool spots [29]. In addition, the

92 extracts were analyzed by PCR for ICP1 and ICP3 (ICP2 PCR technically failed; Table S1).

93 Direct immune-fluorescence was performed as previously described on planktonic cells from

94 RNAlater preserved stool samples [30]. This fraction was obtained by a 15 seconds $100-\mathrm{G}$

95 centrifugation to remove sediment from $500 \mu \mathrm{l}$ of sample, one PBS wash, pelleting the

96 supernatant fraction, and resuspension of the pellet in $500 \mu$ of PBS with $3.7 \%$ formalin. Mock

97 positive control stool samples were used for molecular and microscopy assays that consisted of

98 V. cholerae set to concentrations relative to cholera stool (5e8 CFU/ml and $1 \mathrm{e} 8 \mathrm{CFU} / \mathrm{ml})$ in 500

$99 \mathrm{ml}$ normal saline plus $1.3 \mathrm{ml}$ RNAlater (ratio used in stool storage).

\section{Statistical analysis}

101 Latent class modeling was used to estimate sensitivities and specificities of each diagnostic

102 [31]. For prior information, the assumptions for sensitivities were the same for RDT, qPCR, nl-

103 qPCR and culture (50-100\%). Assumptions for specificities were 50-100\% for RDT, 90-100\% for

104 qPCR and nl-qPCR, and 99-100\% for culture [18]. Gibbs sampling with 100,000 iterations was

105 used to generate posterior estimates with 95\% credible intervals (Cl). Fischer's exact test was

106 used to evaluate associations between diagnostic type and detection of lytic bacteriophage /

107 azithromycin. Both sample odds ratios and estimated sample odds ratios with a conditional

108 Maximum Likelihood Estimate were computed. A two-sample Wilcoxin test was used to

109 compare CT values between diagnostic positive and negative samples among samples positive

110 for $V$. cholerae positive by nl-qPCR CT. Comparison of microbiota (16S rDNA analysis) by

111 diagnostic result and exposure among nl-qPCR positive samples was conducted by

112 PERMANOVA as previously described [21]. Missingness in the dataset is designated as 'NA'

113 and is restricted to laboratory results. Statistical analyses were completed in Graphpad Prism

114 8.0.1 and R v3.4.1/ RStudio v1.1.0153 [32]. 
Data availability

117 Data analyzed in the manuscript have been made available in the online supplementary

118 material.

119 RESULTS

120 Sensitivity and specificity estimates by latent class modeling

121 In Bangladesh, stool samples were collected from 881 of 961 enrolled patients. Among samples

122 tested by RDT, qPCR, and nl-qPCR, the distribution of diagnostic positivity is provided (Fig

123 1A,B). The sensitivities and specificities of each diagnostic were estimated using a Bayesian

124 latent class modeling framework, which enables estimation of diagnostic accuracy in the 125 absence of a perfect reference standard by integrating data from multiple tests [31]. Estimates 126 for sensitivity of RDT, qPCR, and nl-qPCR were 31.5\% (95\% Cl:21.5-43.7), 64.1\% (Cl: 50.7127 80.2) and 97.6\% (95\% Cl: 89.0-100.0), respectively. The specificities were 99.6\% (95\% Cl: 128 99.0-99.9), 99.9\% (95\% Cl: 99.7-100.0) and 99.6\% (95\% Cl: 98.3-100.0), respectively. Among 129 the subset of samples randomly chose for culture (16 positive out of 251 ), sensitivity was $57.1 \%$ 130 (40.4-73.2) and specificity 99.7 (99.3-99.9). Based on these results, nl-qPCR was selected as 131 the best available reference standard for subsequent analysis and the receiver operator curve 132 (ROC) is presented (Fig 1C).

\section{Impact of lytic phage on diagnostic positivity}

134 Among $V$. cholerae positive samples by nl-qPCR, 19.2\% (15/78) and 1.3\% (1/78) were positive

135 for ICP1 and 2, respectively; ICP3 was not detected. Of 180 random samples negative by nl136 qPCR, qPCR and RDT, two patients had ICP1 (one was culture positive) and one had ICP2.

137 Among $V$. cholerae positive samples by nl-qPCR that lacked azithromycin, vibriophage were 138 negatively associated with diagnostic positivity by RDT (OR 0.11; 95\% Cl: 0.002-0.87), qPCR 139 (OR 0.13; 95\% Cl: 0.02-0.65) and direct immuno-fluorescent microscopy [30] (OR 0.18; 95\% Cl $140 \quad 0.02-1.031 ;$ Table 1). Frequencies of vibriophage detection were different between study sites 
141 (Fischer's exact test; $p=0.033$ ). Diarrheal samples from South Sudan were analyzed to

142 increase generalizability [29]. ICP1 was detected in $10.2 \%(n=10 / 98)$ of all enriched samples,

$14324 \%$ of samples $(n=7 / 29)$ that were PCR positive samples for $V$. cholerae and $5.7 \%(n=3 / 69)$ of

144 samples that were RDT negative by PCR for $V$. cholerae. ICP1 was negatively associated with

145 RDT positivity after enrichment (OR 0.00, 95\%Cl 0.00-0.64, p=0.010; Table S2); a statistically

146 significant difference was not observed for unenriched samples. ICP3 was not identified. There

147 were insufficient samples to assess phage impact on culture positivity.

148 Impact of azithromycin on diagnostic positivity

149 Among Bangladesh samples positive by nl-qPCR but negative for bacteriophage, azithromycin

150 was negatively associated with diagnostic positivity by RDT (OR 0.00; 95\% Cl: 0.00-0.28) and

151 qPCR (OR 0.11; 95\% Cl: 0.03-0.44), but not by direct fluorescent microscopy (OR 0.54; $95 \% \mathrm{Cl}$

152 0.14-1.97; Table 2). Azithromycin was negatively associated with culture positivity (OR 0.00,

$15395 \%$ 0.00-0.997; Table 2).

\section{Absolute and relative $V$. cholerae concentration.}

155 Absolute and relative $V$. cholerae concentration was assessed by nl-qPCR and 16S rDNA

156 analysis, respectively. Among $\mathrm{nl-qPCR}$ positive samples, there was a significant inverse

157 relationship between diagnostic positivity and $V$. cholerae concentration (Table S3). With no

158 exclusions, fold-differences between positive and negative samples ranged from 21-fold

159 (culture) to 79-fold (qPCR). The one exception was that phage exposure (azithromycin samples

160 excluded) did not associate with a significant difference in the nl-qPCR Ct values between

161 culture positive $(\mathrm{n}=13 ; \mathrm{Ct}=19.4,95 \% \mathrm{Cl} 14.3-22.0)$ and negative samples $(\mathrm{n}=9 ; \mathrm{Ct}=20.8,95 \%$

162 Cl 17.6-25.9; $p=0.186)$. Statistically significant differences in microbiota (16S rDNA) were

163 observed between RDT positive and negative stools with stratifications for bacteriophage (Fig

164 S2A) and azithromycin (Fig S2B). 


\section{DISCUSSION}

166 This study investigated the potential vulnerability diagnostics have when bacterial targets are

167 exposed to lytic bacteriophage predation or antibiotics. Using cholera as a model system and

$168 \mathrm{nl}-\mathrm{qPCR}$ as a reference standard for $V$. cholerae, we found that the odds of a RDT, qPCR and

169 microscopy diagnostic testing positive were reduced by more than $83 \%$ when lytic

170 bacteriophage were present. Similarly, the odds of a RDT, qPCR and culture testing positive

171 were reduced by more than $89 \%$ when the first-line antibiotic azithromycin was detected in stool

172 by mass spectrometry. These results expose a vulnerability of gold-standard diagnostics that

173 clinicians and microbiologists feared but lacked sufficient data to take evidence-based action.

174 We reason that the low inflection point in the ROC at approximately 0.7 sensitivity is multi-

175 factorial (Fig 1C). We explored the effect on sensitivity and specificity of adding ICP1 detection

176 as a proxy for $V$. cholerae detection (Fig 1C). Both qPCR, culture and the RDT moderately

177 improved. The effects of lytic bacteriophage, antibiotics and host antimicrobial factors on

178 diagnostic positivity are likely additive, especially given that these diagnostics target different

179 biologic mechanisms. How duration of illness and severity of disease serve as determinants of

180 diagnostic positivity also remain unknown. Time-series analyses of cholera patients with defined

181 antimicrobial exposures are needed steps to further these lines of inquiry.

182 These findings should be viewed within the context of the limitations of the study. The

183 procedures were chosen for feasibility at remote field sites. This delayed cultures up to one-

184 month and precluded plaque assays. The higher detection rate of nl-qPCR compared to qPCR

185 was multi-factorial, including the 1.8-fold difference in DNA. The positive nl-qPCR samples that

186 were negative by qPCR and negative by ompW were unlikely to be false positives because

187 Vibrio spp. were detected by $16 \mathrm{~S}$ rDNA analysis in all 13 samples that did not have lytic

188 vibriophage; those with vibriophage did not result $(n=7 / 7)$. Among $n l-q P C R$ positive and $q P C R$ 
189 negative samples, PCR detection for $\operatorname{tpcA}$ correlated with PCR detection of $c t x A$ (cholera toxin;

$190 \mathrm{n}=5 / 5$; Table S1). These toxin data, paired with serologic results that found only $01 \mathrm{~V}$. cholerae,

191 makes the possibility of confounding from non-O1 V. cholerae unlikely. Despite these

192 limitations, the discovery that lytic bacteriophage negatively impacts diagnostics, even to the

193 point that samples will test positive for bacteriophage and negative for the pathogen, has broad

194 significance. One explanation is lytic bacteriophage and antibiotics inhibit bacterial growth below

195 the diagnostic limits of detection. Alternatively, bacteriophage nucleases, or host nucleases

196 responding to bacteriophage infection, may digest host chromosomal DNA to the point that PCR

197 fails $[33,34]$.

198 Conclusion

199 Within the cholera field, this study suggests that more nuanced analytical approaches are

200 needed to determine the true cholera burden during outbreaks, especially in the latter phases

201 when rates of concurrent lytic bacteriophage predation are likely higher $[16,17]$. This may

202 require an approach that includes lytic bacteriophage detection as a proxy for pathogen

203 detection and a de-emphasis on diagnostic results with known antibiotic exposure. Outside the

204 cholera field, these data serve as a call-to-action to survey for lytic bacteriophage when

205 bacterial diagnostics have inconsistent performance, especially when there is discordance

206 between clinical presentation and diagnostic result. These efforts may justify a new line of

207 diagnostic development that targets both the prey (pathogen) and predator (bacteriophage). 


\section{Acknowledgements}

209 We thank the patients for participating in this study, the field and central project staff, and

210 diligent clinical team who made this study possible. We are grateful to M. Barry, S. Luby and Y.

211 Maldonado for their support at Stanford University, as well as G. Morris, A. Ali, M. Alam, E.

212 Schmidt, R. Autrey, K. Berquist and S. Rivkees at the University of Florida for their support,

213 reagents and helpful discussions.

\section{Disclaimer}

215 These funders had no role in study design, data collection and analysis, decision to publish, or 216 preparation of the manuscript.

\section{Financial Support}

218 This work was supported by the National Institutes of Health [DP5OD019893] to EJN,

219 [R01Al123422-01] to DAS and AKD and internal support from the University of Florida and the 220 Stanford University Center for Innovation in Global Health, Translational and Applied Medicine 221 program, and the Child Health Research Institute. DLC was funded by Bill and Melinda Gates 222 through their active support of the Institute for Disease Modeling (Bellevue, WA) and 223 sponsorship through the Global Good Fund.

\section{Disclaimer}

225 These funders had no role in study design, data collection and analysis, decision to publish, or 226 preparation of the manuscript.

\section{Potential conflicts of interest.}

228 All authors: No reported conflicts. 


\section{FIGURE LEGENDS}

231 Figure 1. Diagnostic evaluation. A. Euler diagram of diagnostic positivity for qPCR, nl-qPCR,

232 and RDT; area within each circle is relative to the degree of positivity. B. Comparison Ct values

233 between qPCR and nl-qPCR analysis with ICP1 and ICP2 metadata; horizontal and vertical

234 dotted lines depict thresholds of positivity for each test; ND= not detected. C. Receiver operator

235 characteristic (ROC) curve. Estimates of the sensitivity and 1-specificity of combining

236 diagnostics are defined in the key and vertical bars from each symbol depict the $95 \% \mathrm{Cl}$. 
A.

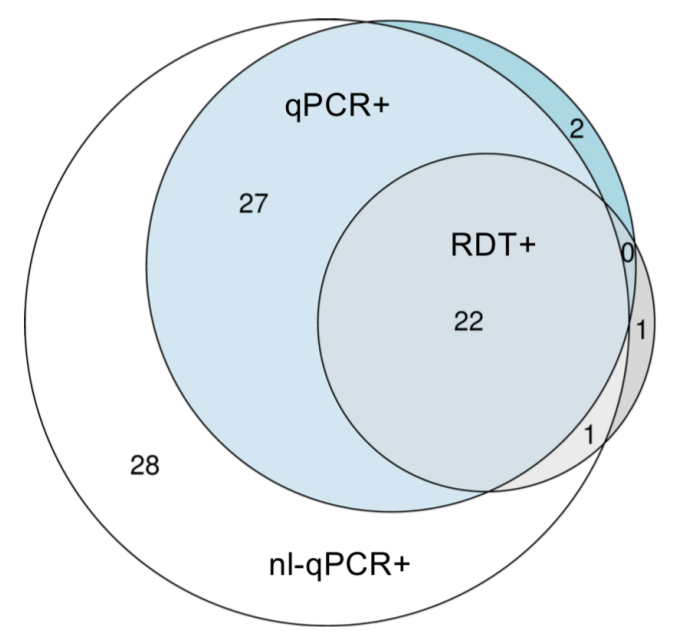

B.

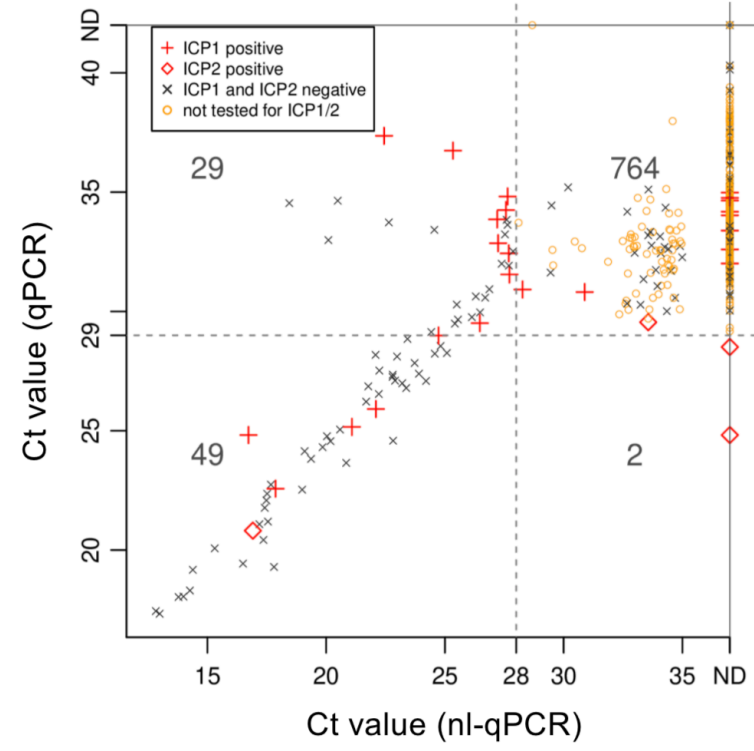

c.

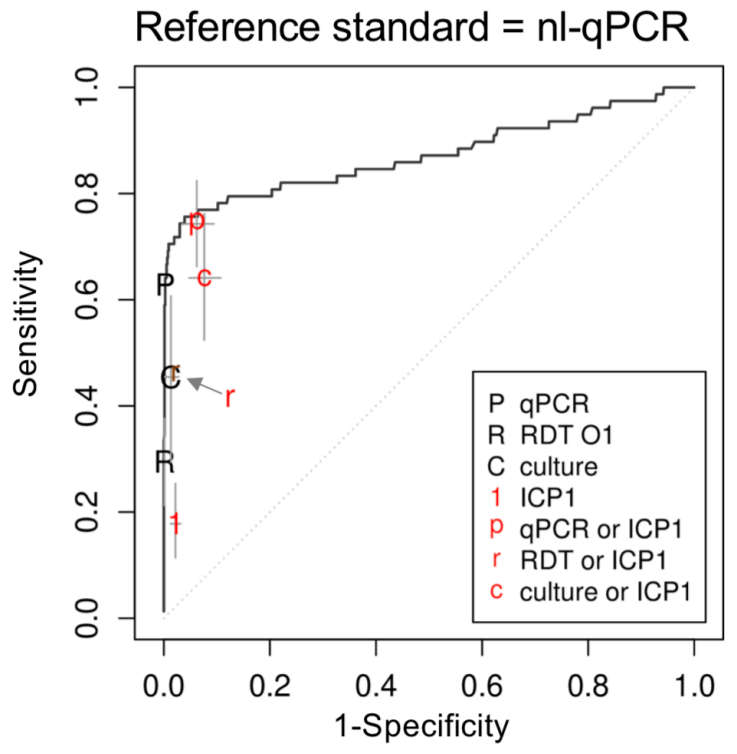

241

243

244

245 
Table 1.

Lytic phage negatively impact diagnostic positivity (azithromycin excluded)

\begin{tabular}{|c|c|c|c|c|c|c|c|}
\hline Diagnostic (Dx) & $\mathrm{N}^{\mathrm{a}}$ & $\begin{array}{l}\text { Dx positive among } \\
\text { phage exposed }\end{array}$ & $\begin{array}{l}\text { Dx positive among } \\
\text { phage unexposed }\end{array}$ & $\mathrm{OR}^{\mathrm{b}}$ & OR_MLE ${ }^{c}$ & $\mathrm{Cl}^{\mathrm{c}}$ & $p^{d}$ \\
\hline RDT & 56 & $9 \%(1 / 11)$ & $49 \%(22 / 45)$ & 0.105 & 0.108 & $0.002-0.872$ & 0.019 \\
\hline qPCR & 56 & $36 \%(4 / 11)$ & $83 \%(37 / 45)$ & 0.124 & 0.130 & $0.022-0.649$ & 0.005 \\
\hline Microscopy ${ }^{e}$ & 52 & $20 \%(2 / 10)$ & $60 \%(25 / 42)$ & 0.170 & 0.176 & $0.016-1.031$ & 0.036 \\
\hline Culture $^{f}$ & 22 & $---(0 / 0)$ & $59 \%(13 / 22)$ & --- & --- & --- & --- \\
\hline
\end{tabular}

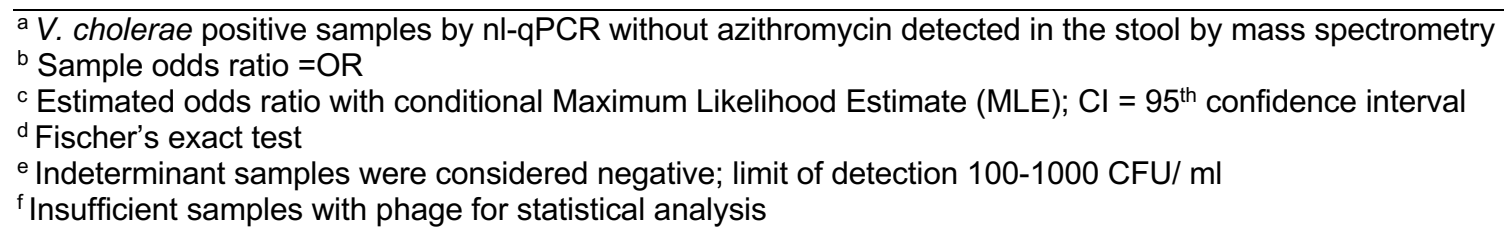

\section{Insert 3 (Table 2)}

Table 2.

265 Azithromycin negatively impacts diagnostic positivity (phage excluded)

\begin{tabular}{lccccccc}
\hline Diagnostic (Dx) & $\mathrm{N}^{\mathrm{a}}$ & $\begin{array}{c}\text { Dx positive among } \\
\text { azithro exposed }\end{array}$ & $\begin{array}{c}\text { Dx positive among } \\
\text { azithro unexposed }\end{array}$ & $\mathrm{OR}^{\mathrm{b}}$ & $\mathrm{OR}_{\mathrm{MLE}}{ }^{\mathrm{C}}$ & $\mathrm{Cl}^{\mathrm{C}}$ & $\mathrm{p}^{\mathrm{d}}$ \\
\hline RDT & 63 & $0 \%(0 / 18)$ & $49 \%(22 / 45)$ & 0.000 & 0.000 & $0.000-0.282$ & $<0.001$ \\
qPCR & 63 & $33 \%(6 / 18)$ & $82 \%(37 / 45)$ & 0.108 & 0.113 & $0.026-0.437$ & $<0.001$ \\
Microscopy e & 58 & $44 \%(7 / 16)$ & $60 \%(25 / 42)$ & 0.529 & 0.535 & $0.139-1.973$ & 0.378 \\
Culture $^{f}$ & 27 & $0 \%(0 / 5)$ & $59 \%(13 / 22)$ & 0.000 & 0.000 & $0.000-0.997$ & 0.041
\end{tabular}

${ }^{a} V$. cholerae positive samples by nl-qPCR without lytic bacteriophage (ICP1,2,3) detected in the stool by PCR

267 b Sample odds ratio $=\mathrm{OR}$

$268{ }^{c}$ Estimated odds ratio with conditional Maximum Likelihood Estimate (MLE); $\mathrm{Cl}=95^{\text {th }}$ confidence interval

269 d Fischer's exact test

270 e Indeterminant samples were considered negative; limit of detection 100-1000 CFU/ ml

271 f Insufficient samples with phage for statistical analysis 


\section{Supplement Material:}

275 Table of contents

276 1. Fig. S1. Diagnostic positivity and ICP1 detection in South Sudan and Bangladesh

277 2. Fig. S2. Microbiota distribution as a function of bacteriophage and azithromycin detection

278 3. Table S1. Reagents

279 4. Table S2. South Sudan: Impact of bacteriophage ICP1 on cholera RDT positivity

280 5. Table S3. Fold-differences in DNA concentration among V. cholerae positive samples by $\mathrm{nl}$

281 qPCR

282 6. Dataset 
A. South Sudan Positivity

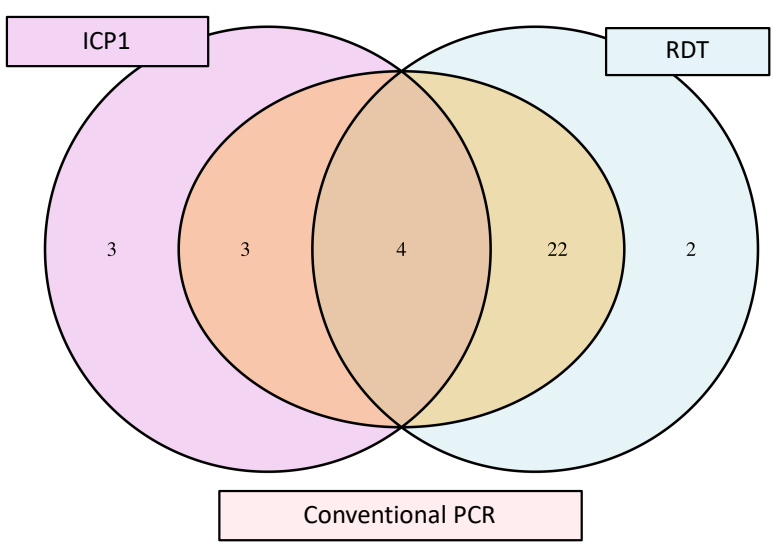

B. Bangladesh Positivity

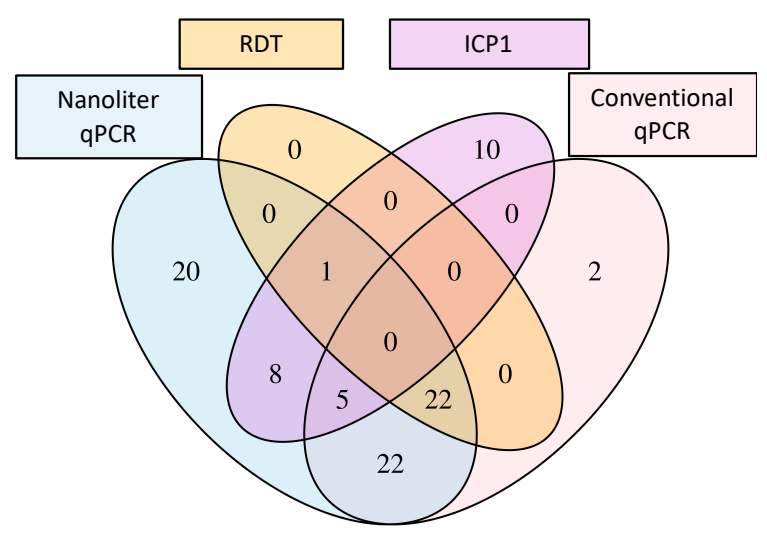

Figure S1. Comparison of diagnostic positivity and ICP1 detection in the libraries from South Sudan (A) and Bangladesh (B). RDT= rapid diagnostic test. Both settings used the Crystal VC test with enrichment. Data presented from S. Sudan is based on PCR performed at Institute Pasteur (Table S2). 

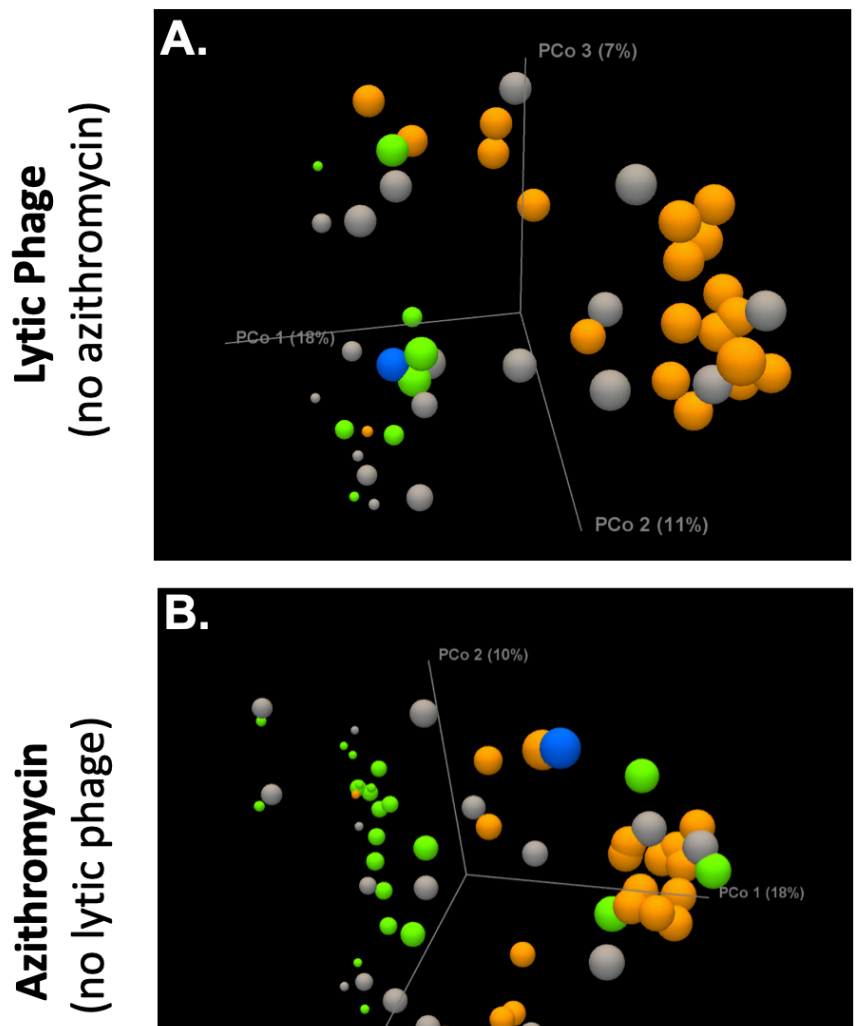

PERMANOVA: Bray-Curtis

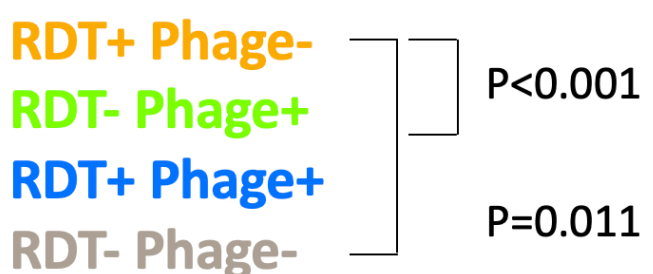

299

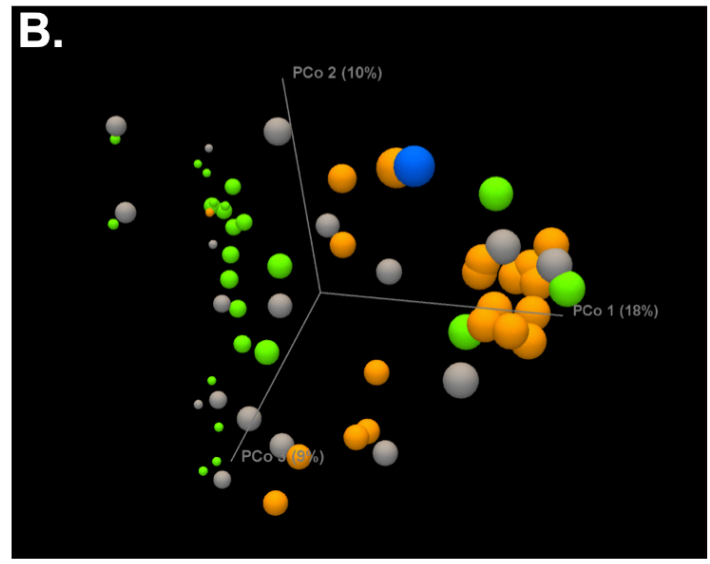

Diameter = Relative Amount (\% by $16 \mathrm{~S})$
PERMANOVA: Bray-Curtis

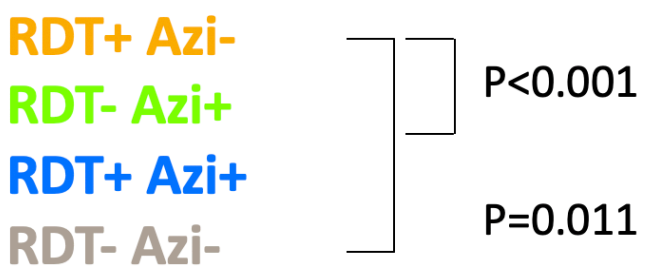

Figure S2. Principal component analysis of $16 \mathrm{~S}$ rDNA analysis of $V$. cholerae positive samples by nl-qPCR analysis as previously described[21]. A. Among samples without azithromycin detection, data are color coded based on RDT and phage positivity (right). B. Among samples without bacteriophage detection, data are color coded based on RDT and azithromycin positivity (right). PC1 is oriented horizontally and the icon size is set to the relative percentage of $V$. cholerae detected in the microbiota $(0-25 \%, 26-50 \%, 51-75 \%, 76-100 \%)$. For both the upper and lower panels, statistically significant differences between groups were detected by PERMANOVA (Bray-Curtis)[21]. V. cholerae positivity is defined by nl-qPCR positivity with either tcpA primer sets to be consistent with prior analytic approach[21]. 


\section{Table S1. Reagents}

\begin{tabular}{|c|c|c|c|}
\hline \multicolumn{4}{|l|}{ Reagent } \\
\hline Bacterium & Strain & Description & Reference \\
\hline V. cholerae & E7946 & O1 serogroup, isolated from Bahrain*, SmR & Mekalanos [35] \\
\hline V. cholerae & ENV2 & Non-O1, from Haiti*, SmS & Rahman et al. [36] \\
\hline V. cholerae & VC037; 280 NAG & Non-O1, from India*, ATCC25872, SmR & Felsenfeld et al. [37] \\
\hline PCR target & Primer name & Sequence $5^{\prime}-3^{\prime}$ & \\
\hline \multirow[t]{2}{*}{$\operatorname{ct} x A$} & ctxA_F & CTCAGACGGGATTTGTTAGGCACG & Hoshino et al. [38] \\
\hline & ctxA_F & TCTATCTCTGTAGCCCCTATTACG & \\
\hline \multirow[t]{2}{*}{ ompW } & ompW_F & CACCAAGAAGGTGACTTTATTGTG & Nandi et al. [39] \\
\hline & ompW_R & GAACTTATAACCACCCGCG & \\
\hline $\operatorname{tcp} A$ & $\begin{array}{l}\text { tcp } A^{\text {set1 }} F \\
\text { tcpA } A^{\text {set1 }} R\end{array}$ & $\begin{array}{l}\text { ACTAAGGCTGCGCAAAATCT } \\
\text { GCCTCATCAGCTGAAACCTT }\end{array}$ & $\begin{array}{c}\text { Grembi \& } \\
\text { Spormann[22] }\end{array}$ \\
\hline $\operatorname{tcp} A$ & $\begin{array}{l}\text { tcpA } A^{\text {set2 }}-F \\
\text { tcpA }{ }^{\text {set2 }} \_^{R}\end{array}$ & $\begin{array}{l}\text { ACACGATAAGAAAACCGGTCA } \\
\text { GCCTTGGTCATATTCTGCGA }\end{array}$ & $\begin{array}{c}\text { Grembi \& } \\
\text { Spormann[22] }\end{array}$ \\
\hline ICP1 & $\begin{array}{l}\text { ICP1gp58F } \\
\text { ICP1gp58R }\end{array}$ & $\begin{array}{l}\text { AACGCTGCTTTTCCTTTTGA } \\
\text { CCCAGCATTGAGGACACTT }\end{array}$ & Seed et al. [14] \\
\hline ICP2 & $\begin{array}{l}\mathrm{ICP} 2 \_4 \mathrm{~F} \\
\mathrm{ICP} 2 \_4 \mathrm{R}\end{array}$ & $\begin{array}{l}\text { CGCTAGTTCTGGCAGTGA GT } \\
\text { TCCGTTCCAGTTCCAACAGG }\end{array}$ & Alexandrova et al.[21] \\
\hline ICP2 & $\begin{array}{l}\text { ICP2_24R } \\
\text { ICP2_24R }\end{array}$ & $\begin{array}{l}\text { AGAAGTCGCAAACGGGGTAC } \\
\text { AACGTGGTTCTCGTGAGTGG }\end{array}$ & Alexandrova et al.[21] \\
\hline ICP3 & $\begin{array}{l}\text { ICP3gp5F } \\
\text { ICP3gp5F }\end{array}$ & $\begin{array}{l}\text { ATTGTCGAGTGGGACAAAGG } \\
\text { ACCAACTCGACGCATAGCTT }\end{array}$ & Seed et al. [14] \\
\hline $16 S$ rDNA ${ }^{* *}$ & Maeda_1048_1067_F & GTGSTGCAYGGYTGTCGTCA & Maeda et al. [40] \\
\hline $16 S$ rDNA ${ }^{* *}$ & $\begin{array}{l}\text { Maeda_1175_1194_R } \\
\text { 27F_Miseq } \\
\text { 338rcbc1 }\end{array}$ & $\begin{array}{l}\text { ACGTCRTCCMCACCTTCCTC } \\
\text { AATGATACGGCGACCACCGAGATCTACA } \\
\underline{C} \text { CATGGTAATTCCAGMGTTYGATYMTGG } \\
\text { CTCAG } \\
\text { CAAGCAGAAGACGGCATACGAGAT } \\
\text { ACGAGACTGATTAGTCAGTCAGaaGCTG } \\
\text { CCTCCCGTAGGAGT }\end{array}$ & Chung et al. [41] \\
\hline $\begin{array}{l}\text { *Strain used a } \\
\text { ** } 16 \mathrm{~S} \text { rDNA p } \\
\text { (http://arep.m } \\
\text { microbiome a } \\
\text { lllumina adapt } \\
\text { reverse prime } \\
\text { Reverse prim }\end{array}$ & $\begin{array}{l}\text { a control strain for testing } \\
\text { imer pair used for nanoliter } \\
\text { d.harvard.edu/labgc/adnan/ } \\
\text { alysis. Degenerate primers } \\
\text { er, (ii) Forward primer pad, } \\
\text { example: (i) Reverse comp } \\
\text { r pad (italics), (iv) Reverse }\end{array}$ & $\begin{array}{l}\text { pecificity of } t c p A \text { primers set } 1 \text { and set2. } \\
\text { PCR. Degenerate primers are coded per standard } \\
\text { rojects/Utilities/revcomp.html). Example of } 16 \mathrm{~S} \text { rDN } \\
\text { are coded per standard convention. Structure of forv } \\
\text { ii) Forward primer linker (lower case), (iv) Forward p } \\
\text { ement of } 3^{\prime} \text { lllumina adapter (underlined), (ii) Golay } \\
\text { rimer linker (lower case), (v) Reverse primer. }\end{array}$ & $\begin{array}{l}\text { Anvention } \\
\text { A primer pair used for } \\
\text { ard primer: (i) } \underline{5^{\prime}} \\
\text { imer. Structure of } \\
\text { arcode (bold text), (iii) }\end{array}$ \\
\hline
\end{tabular}


321 Table S2. South Sudan: Impact of bacteriophage ICP1 on cholera RDT positivity

\begin{tabular}{|c|c|c|c|c|c|c|c|c|}
\hline & & Diagnost & Positive & Diagnostic & Negative & & & \\
\hline Diagnostic Test & Total & Phage Pos & Phage Neg & Phage Pos & Phage Neg & OR_MLE ${ }^{a}$ & $\mathrm{Cl}^{\mathrm{a}}$ & $\mathrm{P}^{\mathrm{b}}$ \\
\hline \multicolumn{9}{|l|}{$\begin{array}{l}\text { Among VC PCR } \\
\text { positive (Hopkins) }\end{array}$} \\
\hline RDT (Direct) ${ }^{d}$ & 32 & 7 & 26 & 1 & 0 & --- & --- & --- \\
\hline RDT (Enriched) & 34 & 5 & 25 & 3 & 1 & 0.075 & $0.001-1.137$ & 0.033 \\
\hline \multicolumn{9}{|l|}{$\begin{array}{l}\text { Among VC PCR } \\
\text { positive (Pasteur) }\end{array}$} \\
\hline RDT (Direct) ${ }^{d}$ & 29 & 6 & 22 & 1 & 0 & --- & --- & --- \\
\hline RDT (Enriched) & 29 & 4 & 22 & 3 & 0 & 0.000 & $0.000-0.641$ & 0.010 \\
\hline
\end{tabular}

324 c Conventional PCR was performed by Johns Hopkins University and Institute Pasteur for cross validation [29].

325 'Direct' = tested directly from stool; 'Enriched' = grown in selective media (APW) prior to testing. Values reported in

326 the main text and Figure S1 are derived from the Institute Pasteur data set.

327 d Insufficient sample size for statistical analysis 
Table S3.

Fold-differences in target DNA detection among $V$. cholerae positive samples

\begin{tabular}{|c|c|c|c|c|c|}
\hline & & Diagnostic Positive & Diagnostic Negative & & \\
\hline Diagnostic Test & Total $^{1}$ & Median nl-qPCR Ct (n; IQR) & Median nl-qPCR Ct (n; IQR) & Fold-difference & $\mathrm{p}^{2}$ \\
\hline \multicolumn{6}{|l|}{ No exclusion } \\
\hline RDT & 78 & $19.0(23 ; 16.5-21.7)$ & $24.4(55 ; 21.1-27.7)$ & 42 & $<0.001$ \\
\hline qPCR & 78 & $20.2(49 ; 17.4-22.9)$ & $26.5(29 ; 24.9-27.6)$ & 79 & $<0.001$ \\
\hline Microscopy ${ }^{3}$ & 71 & $20.3(34 ; 17.3-23.0)$ & $25.1(37 ; 21.5-27.3)$ & 28 & $<0.001$ \\
\hline Culture & 29 & $19.4(13 ; 14.3-22.0)$ & $23.8(16 ; 17.7-25.5)$ & 21 & 0.013 \\
\hline \multicolumn{6}{|c|}{ Includes samples with azithromycin (excludes phage samples) } \\
\hline RDT & 63 & $18.4(22 ; 16.2-21.1)$ & $23.9(41 ; 21.3-26.2)$ & 46 & $<0.001$ \\
\hline qPCR & 63 & $20.2(43 ; 17.4-22.9)$ & $26.2(20 ; 24.5-27.5)$ & 65 & $<0.001$ \\
\hline Microscopy ${ }^{3}$ & 58 & $20.3(32 ; 17.4-23.2)$ & $24.5(26 ; 20.7-26.2)$ & 18 & 0.002 \\
\hline Culture & 27 & $19.4(13 ; 14.3-22.0)$ & $23.8(14 ; 17.8-25.5)$ & 21 & 0.011 \\
\hline \multicolumn{6}{|c|}{ Includes samples with phage (excludes azithromycin samples) } \\
\hline RDT & 56 & $19.0(23 ; 16.5-21.7)$ & $24.6(33 ; 21.1-27.0)$ & 48 & $<0.001$ \\
\hline qPCR & 56 & $19.9(41 ; 17.4-22.8)$ & $27.2(15 ; 25.4-27.6)$ & 163 & $<0.001$ \\
\hline Microscopy ${ }^{3}$ & 52 & $19.1(27 ; 17.2-22.8)$ & $24.8(25 ; 21.5-27.2)$ & 53 & $<0.001$ \\
\hline Culture & 22 & $19.4(13 ; 14.3-22.0)$ & $20.8(09 ; 17.6-25.9)$ & 2.8 & 0.186 \\
\hline
\end{tabular}

$328 \quad{ }^{1}$ Nanoliter $(\mathrm{nL})$ qPCR positive for $\mathrm{V}$. cholerae $(\mathrm{Ct}<28)$; a random subset were cultured or available for microscopy.

3292 Mann-Whitney U test (two-tailed)

$330{ }^{3}$ Indeterminant samples scored as negative; limit of detection 100-1000 CFU/ ml 


\section{References}

333 [Main text and supplement references are combined]

1. Global Burden of Disease Collaborators. Estimates of the global, regional, and national morbidity, mortality, and aetiologies of diarrhoea in 195 countries: a systematic analysis for the Global Burden of Disease Study 2016. Lancet Infect Dis 2018; 18:1211-28.

2. Ali M, Nelson AR, Lopez AL, Sack DA. Updated global burden of cholera in endemic countries. PLoS Negl Trop Dis 2015; 9:e0003832.

3. Nelson EJ, Harris JB, Morris JG, Jr., Calderwood SB, Camilli A. Cholera transmission: the host, pathogen and bacteriophage dynamic. Nat Rev Microbiol 2009; 7:693-702. 4. D'Herelle F, Malone R. A preliminary report of work carried out by the cholera bacteriophage enquiry. Indian Medical Gazette 1927:614-7.

343 5. Kaper JB, Morris JG, Jr., Levine MM. Cholera. Clin Microbiol Rev 1995; 8:48-86. 6. Musekiwa A, Volmink J. Oral rehydration salt solution for treating cholera: $</=270 \mathrm{mOsm} / \mathrm{L}$ solutions vs >/= $310 \mathrm{mOsm} / \mathrm{L}$ solutions. Cochrane Database Syst Rev 2011:CD003754. 7. Leibovici-Weissman Y, Neuberger A, Bitterman R, Sinclair D, Salam MA, Paul M. Antimicrobial drugs for treating cholera. Cochrane Database Syst Rev 2014:CD008625.

8. Nelson EJ, Nelson DS, Salam MA, Sack DA. Antibiotics for both moderate and severe cholera. N Engl J Med; 364:5-7.

9. Arifuzzaman M, Ahmed T, Rahman MA, et al. Individuals with Le(a+b-) blood group have increased susceptibility to symptomatic Vibrio cholerae 01 infection. PLoS Negl Trop Dis 2011;

$352 \quad 5: e 1413$.

353 10. Merrell DS, Butler SM, Qadri F, et al. Host-induced epidemic spread of the cholera bacterium. Nature 2002; 417:642-5. 11. Nelson EJ, Chowdhury A, Flynn J, et al. Transmission of Vibrio cholerae is antagonized by lytic phage and entry into the aquatic environment. PLoS Pathog 2008; 4:e1000187. 12. Hartley DM, Morris JG, Jr., Smith DL. Hyperinfectivity: a critical element in the ability of Vibrio cholerae to cause epidemics? PLoS Med 2006; 3:e7. 13. Seed KD, Yen M, Shapiro BJ, et al. Evolutionary consequences of intra-patient phage predation on microbial populations. Elife 2014; 3:e03497. 14. Seed KD, Bodi KL, Kropinski AM, et al. Evidence of a dominant lineage of Vibrio choleraespecific lytic bacteriophages shed by cholera patients over a 10-year period in Dhaka, Bangladesh. MBio 2011; 2:e00334-10. 15. Jensen MA, Faruque SM, Mekalanos JJ, Levin BR. Modeling the role of bacteriophage in the control of cholera outbreaks. Proc Natl Acad Sci U S A 2006; 103:4652-7. with the prevalence of environmental cholera phages. Proc Natl Acad Sci U S A 2005; 102:17027.

17. Faruque SM, Islam MJ, Ahmad QS, et al. Self-limiting nature of seasonal cholera epidemics: Role of host-mediated amplification of phage. Proc Natl Acad Sci U S A 2005; 102:6119-24. 18. Page AL, Alberti KP, Mondonge V, Rauzier J, Quilici ML, Guerin PJ. Evaluation of a rapid test for the diagnosis of cholera in the absence of a gold standard. PLoS One 2012; 7:e37360. 
19. Dick MH, Guillerm M, Moussy F, Chaignat CL. Review of two decades of cholera diagnostics-how far have we really come? PLoS Negl Trop Dis 2012; 6:e1845. 20. Hasan JA, Bernstein D, Huq A, Loomis L, Tamplin ML, Colwell RR. Cholera DFA: an improved direct fluorescent monoclonal antibody staining kit for rapid detection and enumeration of Vibrio cholerae 01. FEMS Microbiol Lett 1994; 120:143-8. 21. Alexandrova L, Haque F, Rodriguez P, et al. Identification of widespread antibiotic exposure in cholera patients correlates with clinically relevant microbiota changes. J Infect Dis 2019. 22. Grembi J, Mayer-Blackwell K, Luby S, Spormann A. High-throughput multi-parallel enteropathogen quantification via nano-liter qPCR. Available at: bioRxiv 2019 746446; doi: https://doi.org/10.1101/746446 23. Debes AK, Ateudjieu J, Guenou E, et al. Clinical and Environmental Surveillance for Vibrio cholerae in Resource Constrained Areas: Application During a 1-Year Surveillance in the Far North Region of Cameroon. Am J Trop Med Hyg 2016; 94:537-43. 24. George CM, Rashid MU, Sack DA, et al. Evaluation of enrichment method for the detection of Vibrio cholerae $\mathrm{O} 1$ using a rapid dipstick test in Bangladesh. Trop Med Int Health 2014. 25. Ley B, Khatib AM, Thriemer K, et al. Evaluation of a rapid dipstick (Crystal VC) for the diagnosis of cholera in Zanzibar and a comparison with previous studies. PLoS One 2012; 7:e36930. 26. Alam M, Hasan NA, Sultana M, et al. Diagnostic limitations to accurate diagnosis of cholera. J Clin Microbiol 2010; 48:3918-22.

27. Tuteja U, Kumar S, Shukla J, Kingston J, Batra HV. Simultaneous direct detection of toxigenic and non-toxigenic Vibrio cholerae from rectal swabs and environmental samples by sandwich ELISA. J Med Microbiol 2007; 56:1340-5.

28. Haque F, Ball RL, Khatun S, et al. Evaluation of a Smartphone Decision-Support Tool for Diarrheal Disease Management in a Resource-Limited Setting. PLoS NegI Trop Dis 2017; 11:e0005290. Diagnostic Performance Equivalent to Culture. PLoS One 2016; 11:e0168257. 30. Nelson EJ, Chowdhury A, Harris JB, et al. Complexity of rice-water stool from patients with Vibrio cholerae plays a role in the transmission of infectious diarrhea. Proc Natl Acad Sci U S A 2007; 104:19091-6.

404 31. Islam K, Sayeed MA, Hossen E, et al. Comparison of the Performance of the TPTest, Tubex, Typhidot and Widal Immunodiagnostic Assays and Blood Cultures in Detecting Patients with Typhoid Fever in Bangladesh, Including Using a Bayesian Latent Class Modeling Approach. PLoS Negl Trop Dis 2016; 10:e0004558. Statistical Computing. Available at: http://www.R-project.org/. Accessed Dec 29, 2019. 33. McKitterick AC, Hays SG, Johura FT, Alam M, Seed KD. Viral Satellites Exploit Phage Proteins to Escape Degradation of the Bacterial Host Chromosome. Cell Host Microbe 2019; 26:504-14 e4. defective in the ability to degrade host deoxyribonucleic acid. J Virol 1970; 5:700-8. 35. Mekalanos JJ. Duplication and amplification of toxin genes in Vibrio cholerae. Cell 1983; 35:253-63. 
417 36. Rahman M, Jubair M, Alam MT, et al. High-frequency rugose exopolysaccharide production

418 by Vibrio cholerae strains isolated in Haiti. PLoS One 2014; 9:e112853.

419 37. Felsenfeld O, Stegherr-Barrios A, Aldova E, Holmes J, Parrott MW. In vitro and in vivo studies 420 of streptomycin-dependent cholera vibrios. Appl Microbiol 1970; 19:463-9.

421 38. Hoshino K, Yamasaki S, Mukhopadhyay AK, et al. Development and evaluation of a multiplex 422 PCR assay for rapid detection of toxigenic Vibrio cholerae 01 and O139. FEMS Immunol Med 423 Microbiol 1998; 20:201-7.

424 39. Nandi B, Nandy RK, Mukhopadhyay S, Nair GB, Shimada T, Ghose AC. Rapid method for 425 species-specific identification of Vibrio cholerae using primers targeted to the gene of outer 426 membrane protein OmpW. J Clin Microbiol 2000; 38:4145-51.

427 40. Maeda H, Fujimoto C, Haruki Y, et al. Quantitative real-time PCR using TaqMan and SYBR 428 Green for Actinobacillus actinomycetemcomitans, Porphyromonas gingivalis, Prevotella 429 intermedia, tetQ gene and total bacteria. FEMS Immunol Med Microbiol 2003; 39:81-6.

430 41. Chung WS, Walker AW, Louis $P$, et al. Modulation of the human gut microbiota by dietary 431 fibres occurs at the species level. BMC Biol 2016; 14:3. 